\title{
Corticosteroid- modified dermatophytosis- a unique presentation
}

\section{Introduction}

Dermatophytosis is a superficial fungal infection of keratinized tissue (skin, hairs and nails) by dermatophytes. It is caused by the three genera of dermatophytes: Trichophyton, Epidermophyton and Microsporum. According to their habitat and source of infection, it can be anthropophilic, zoophilic and geophilic. Anthropophilic dermatophytes cause human-to-human infection and causes less inflammation. Zoophilic dermatophytes cause infection in animals, but can be transmitted to humans and are characterized by acute inflammatory lesions. Geophilic dermatophytes are found in soil and are inflammatory in nature.

According to the new taxanomy, dermatophyte species are grouped into seven genera: Arthroderma, Trichophyton, Epidermophyton, Nannizzia, Paraphyton, Microsporum, Lophophyton. ${ }^{1}$

The most common clinical form seen in India is tinea corporis, followed by tinea cruris. ${ }^{2}$ In the recent article by Pietro Nen off et al., titled 'The current Indian epidemic of superficial dermatophytosis due to Trichophyton mentagrophytes - a molecular study', has revealed a solely occurring species T. mentagrophytes, genotype VIII. ${ }^{3}$

Recently, inadvertent use of corticosteroids; both topical and systemic, has resulted in atypical, extensive and recalcitrant dermatophytic infection. Also, genital dermatophytosis is being reported with increased frequency. ${ }^{4-11}$ Here we are reporting an unusual presentation of corticosteroid- modified tinea genitalis in a male patient.

Keywords: triamcinolone injection, septate fungal hyphae, macroconidia, antifungal therapy

Abbrevations: LPCB, lactophenol cotton blue; OTC, over-thecounter; FDC, fixed-dose-combination

\section{Case report}

A 42 years old male came to our Skin OPD with complaints of severe itching and redness in the groin and genitalia region since 6 months. He went to an un-qualified medical practitioner who prescribed him salicylic acid topically (Figure 1A) and intra-muscular triamcinolone injection (Figure 1B). After taking this medication, the condition worsened.
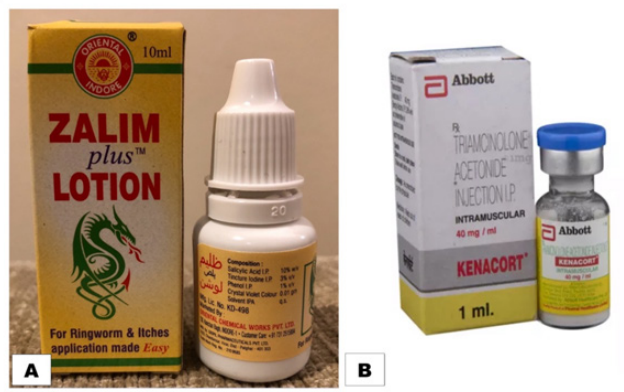

Figure I (A) Lotion containing 10\% salicylic acid. (B) Intramuscular triamcinolone injection $40 \mathrm{mg} / \mathrm{mL}$.
Volume 3 Issue 4 - 2019

\author{
Avneet Singh Kalsi,' Rameshwari Thakur, ${ }^{2}$ \\ Pragya Kushwaha ${ }^{3}$ \\ Muzaffarnagar Medical College, India
}

\begin{abstract}
Correspondence: Avneet Singh Kalsi, Muzaffarnagar Medical College, Muzaffarnagar, Uttar Pradesh, India
\end{abstract} Email avneet.singh.kalsi@gmail.com

Received: July 22, 2019 | Published: July 30, 2019

On physical examination, there were extensive, scaly and thick plaques over the penile shaft, along with preputial involvement and scrotum (Figure 2A) and extended upto abdomen (Figure 2B), which is known as tinea cruris et corporis. Patient was sent to the microbiology laboratory for the collection and processing of the sample.

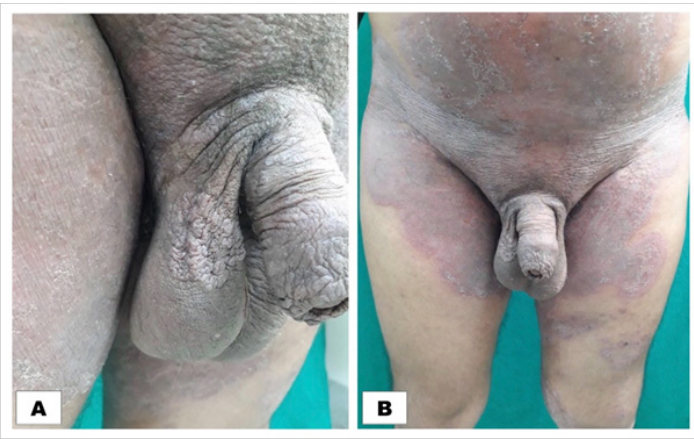

Figure 2 (A) Extensive erythematous, lichenified scaly plaques involving scrotum and prepuce. (B) Lesions extending upto abdomen.

The sample was collected from the lesions only after thoroughly cleansing the site using $70 \%$ ethyl alcohol. The skin scrapings were collected using sterile scalpel blade from the active borders directly in a sterile petri dish. The sample was subjected to potassium hydroxide (KOH) $20 \%$ mount under high power of the microscope which revealed branching septate hyphae. The sample was then inoculated on Sabouraud's Cycloheximide Chloramphenicol Agar (HiMedia). The petri plate was incubated for a period of four weeks at $25^{\circ} \mathrm{C}$ and was examined every week for the evidence of fungal growth. Colonies of Trichophyton mentagrophytes were powdery to fluffy-cottony, cream to white on obverse and beige to brown on reverse. Lactophenol Cotton Blue (LPCB) mounts by teased mount and scotch tape method were prepared which showed septate fungal hyphae, with numerous spherical microconidia arranged in grape-like clusters, cigar-shaped macroconidia and spiral hyphae. Some other tests like urease and in vitro hair perforation test were done for the differentiation of $T$. mentagrophytes and T. rubrum.

The patient was prescribed oral tablets of terbinafine $250 \mathrm{mg}$ once daily for four weeks and $1 \%$ topical luliconazole cream for six weeks. He was recommended to avoid prolonged exposure to moisture and to keep the affected area dry. 
He showed gradual improvement with reduction in crusting and induration and complete resolution after six weeks.

\section{Summary}

Earlier, superficial dermatophytosis was considered as a minor disease which was easier to treat, but now it has acquired an epidemic proportions in India because of misuse of unregulated combination of steroid. ${ }^{7}$ Dermatologists in India are seeing an epidemic of difficult to treat, recalcitrant, recurrent and chronic dermatophytosis over the last 5-6 years. ${ }^{12-14}$ Treating such lesions poses an extraordinary challenge to the dermatologists.

Population of India increased by 3.35 times since independence (1947). 68.86\% Indian lives in rural areas and $31.14 \%$ lives in urban areas. ${ }^{15}$ People in rural areas do not have access to dermatologists, so either they consult an un-qualified medical practitioner or they buy over-the-counter (OTC) fixed-dose-combination (FDC) creams containing two or more active drugs in fixed-dose ratio. Studies have revealed that $59.3 \%$ urban patients and $96 \%$ rural patients have used topical steroid creams without a doctor's recommendation. ${ }^{16}$

The 85 percent of the Indian pharmaceutical market involves "corticosteroid-mixed concoctions", which are fixed portion blends of topical corticosteroids and a couple of antimicrobials and antifungals. ${ }^{17}$ The most common combination is ofloxacin+ornidazole+terbinafine hydrochloride+clobetasol propionate cream (Figure 3).

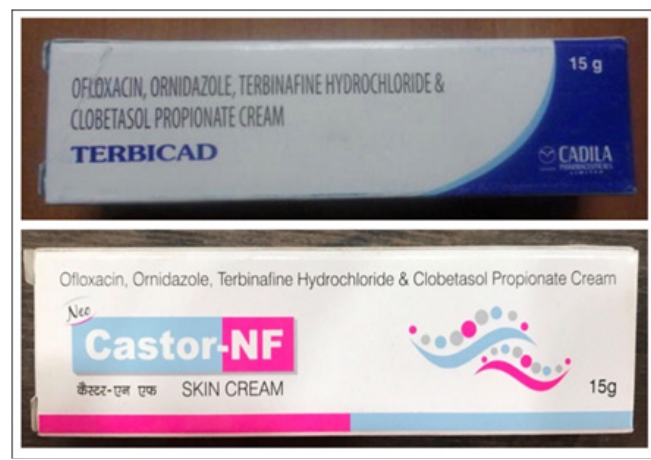

Figure 3 Fixed Drug Combinations (FDC) creams containing a corticosteroid, antifungal and antibacterial with three to five molecules in a product.

Diagnosis of the causative dermatophyte is significant so as to treat and control the infection. The diagnosis of tinea genitalis cannot be made without proper examination of the patient. The whole extent of the disease is noticeable when a patient's thighs are abducted and flexed. It is then perceived that how remarkably exempts the penis and scrotum remain. ${ }^{18}$

Association of glans penis is considered even rarer. ${ }^{19}$ The low frequency of scrotal dermatophytosis, even in the presence of extensive involvement of groin and thighs, has been credited to capric acid in the epidermal barrier, especially abundant in scrotal skin, thought to act as an antifungal factor. ${ }^{20}$ The uncommonness of contamination of penile shaft could be because of diminished eccrine perspiration secretions bringing about diminished hydration of the penile shaft. ${ }^{21}$

Teamwork of dermatologist, mycologists, pharmacists, drug regulatory authorities, government and patients is very essential to curb this disease. Fungal culture ought to be done after clinical cure following the course of the antifungal treatment to affirm mycological cure. Treatment isn't in every case simple, particularly when the patients have utilized topical corticosteroids. Longer time of antifungal treatment with higher portions are required and ought to be tapered gradually. ${ }^{22}$

The treatment comprises of antifungal therapy such a terbinafine $250 \mathrm{mg}$ /day for 2-4 weeks, Itraconazole $200-400 \mathrm{mg} /$ day for 1-2 weeks, combined with topical antifungal creams such as $1 \%$ terbinafine cream once daily application or $1 \%$ luliconazole cream.

\section{Acknowledgments}

None.

\section{Funding}

None.

\section{Conflicts of interest}

Author declares that there is no conflict of interest.

\section{References}

1. de Hoog GS, Dukik K, Monod M, et al. Toward a Novel Multilocus Phylogenetic Taxonomy for the Dermatophytes. Mycopathologia. 2017;182(1-2):5-31.

2. Surendran KAK, Bhat RM, Boloor R, et al. A clinical and mycological study of dermatophytic infections. Indian J Dermatol. 2014;59(3):262267.

3. NenoffP, VermaSB, Vasani R, etal. The current Indian epidemic of superficial dermatophytosis due to Trichophyton mentagrophytes - a molecular study. Mycoses. 2019;62(4):336-356.

4. Verma S, Madhu R. The great Indian epidemic of superficial dermatophytosis: An appraisal. Indian J Dermatol. 2017;62(3):227-236.

5. Panda S, Verma S. The menace of dermatophytosis in India: The evidence that we need. Indian J Dermatol Venereol Leprol. 2017;83(3):281-284.

6. Kupsch C, Czaika VA, Deutsch C, et al. Trichophyton mentagrophytes a new genotype of zoophilic dermatophyte causes sexually transmitted infections. J Dtsch Dermatol Ges. 2019;17(5):493-501.

7. Verma SB, Vasani R. Male genital dermatophytosis - Clinical features and the effects of the misuse of topical steroids and steroid combinations - An alarming problem in India. Mycoses. 2016;59(10):606-614.

8. Thakur R, Kushwaha P, Kalsi AS, et al. Tinea genitalis in a tertiary care hospital of Western U.P. Indian J Clin Exp Dermatol. 2018;4(4):266-273.

9. Prohić, A, Krupalija-Fazlić M, Jovović Sadiković T. Incidence and etiological agents of genital dermatophytosis in males. Med Glas (Zenica). 2015;12(1):52-56.

10. Thakur R, Kushwaha P, Kumar H, et al. Tinea crurisand Tinea genitalis due to Trichophyton interdigitale in and around Muzaffarnagar (Western UP), India: Possibly an Outbreak. IntJ Curr Microbiol App Sci. 2016;5(9):468473 .

11. Luchsinger I, Bosshard PP, Kasper RS, et al. Tinea genitalis: a new entity of sexuality transmitted infection? Case series and review of literature. Sex Transm Infect. 2015;91(7):493-496.

12. Rengasamy M, Chellam J, Ganpati S. Systemic Therapy of Dermatophytosis: Practical and Systemic approach. Clin Dermatol Rev. 2017;1(3):19-23

13. Thakur R, Kalsi AS, Kushwaha P, et al. Epidemiology of cortico-steroidmodified tinea: study of 100 cases in a rural tertiary care teaching hospital of Western Uttar Pradesh, India. J DermatCosmetol. 2018;2(5):64-69. 
14. Kalsi AS, Thakur R, Kushwaha P. Extensive tinea corporis and tinea cruriset corporis due to Trichophyton interdigitale. J DermatCosmetol. 2019;3(1):16-20.

15. Population of India, Ministry of Statistics and Programme Implementation. 2019.

16. Saraswat A, Lahiri K, Chatterjee M, et al. Topical corticosteroid abuse on the face: A prospective, multicentre study of dermatology outpatients. Indian J Dermatol Venereol Leprol. 2011;77(2):160-166.

17. Verma SB. Topical corticosteroid misuse in India is harmful and out of control. BMJ. 2015;351:h6079.

18. Hebra F. Handbuch der speciellen Pathologic and Therapie. Virchow R, Erlagen Ed, Enke III, 1860-31. Hilton-Fagge and Pye-smith PH, editors In: On Disorders of skin. London, New-Syndenham Society, I La Touche CJ. Scrotal dermatophytosis: an insufficient documented aspect of Tinea cruris. Brit J Dermatol.1967;79(6):339-344.
19. D'Antuono A, Bardazzi F, Andalou F. Unusual manifestations of dermatophytosis. Int J Dermatol.2001;40:164-166.

20. Smith JG, Fisher RW, Blank H. The epidermal barrier: a comparison between scrotal and abdominal skin. J Invest Dermatol. 1961;36(5):337344 .

21. Pillai KG, Singh G, Sharma BM. Trichophyton rubrum infection of the penis. Dermatologica. 1975;150:252-254.

22. Dogra S,Uprety S. The menace of chronic and recurrent dermatophytosis in India: Is the problem deeper than we perceive? Indian Dermatol Online J. 2016;7(2):73-76. 